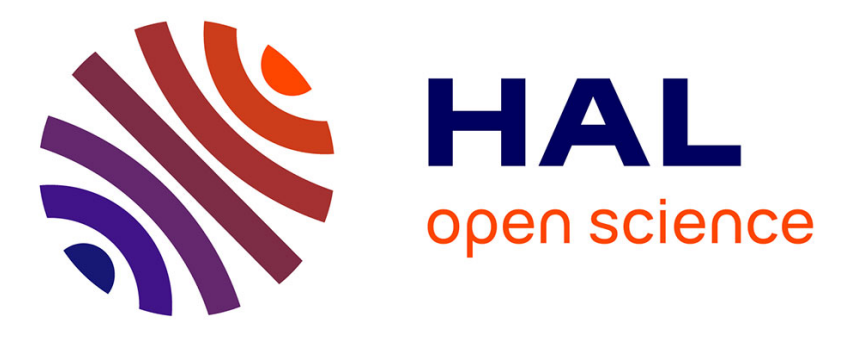

\title{
Investigation of the effects of two major secretory granules components, insulin and zinc, on human-IAPP amyloid aggregation and membrane damage
}

Lucie Khemtemourian, Federico Antoniciello, Bikash R Sahoo, Marion

Decossas, Sophie Lecomte, Ayyalusamy Ramamoorthy

\section{To cite this version:}

Lucie Khemtemourian, Federico Antoniciello, Bikash R Sahoo, Marion Decossas, Sophie Lecomte, et al.. Investigation of the effects of two major secretory granules components, insulin and zinc, on human-IAPP amyloid aggregation and membrane damage. Chemistry and Physics of Lipids, 2021, 10.1016/j.chemphyslip.2021.105083 . hal-03376343

\section{HAL Id: hal-03376343 \\ https://hal.science/hal-03376343}

Submitted on 13 Oct 2021

HAL is a multi-disciplinary open access archive for the deposit and dissemination of scientific research documents, whether they are published or not. The documents may come from teaching and research institutions in France or abroad, or from public or private research centers.
L'archive ouverte pluridisciplinaire HAL, est destinée au dépôt et à la diffusion de documents scientifiques de niveau recherche, publiés ou non, émanant des établissements d'enseignement et de recherche français ou étrangers, des laboratoires publics ou privés. 
Investigation of the effects of two major secretory granules components, insulin and zinc, on humanIAPP amyloid aggregation and membrane damage

Lucie Khemtemourian*a, Federico Antoniciello ${ }^{\mathrm{a}}$, Bikash R. Sahoo ${ }^{\mathrm{b}}$, Marion Decossas ${ }^{\mathrm{a}}$, Sophie Lecomte ${ }^{\mathrm{a}}$ and Ayyalusamy Ramamoorthy* ${ }^{\mathrm{b}}$

${ }^{a}$ CBMN, CNRS UMR 5248, IPB, Univ. Bordeaux, F-33600 Pessac, France. lucie.khemtemourian@ubordeaux.fr

${ }^{\mathrm{b}}$ Biophysics Program, Department of Chemistry, Biomedical Engineering, and Macromolecular Science and Engineering, The University of Michigan, Ann Arbor, MI 48109-1055, USA. ramamoor@ umich.edu

Chemistry and Physics of Lipids, special issue on "Lipids on Pathomechanisms of Amyloid Diseases"

\begin{abstract}
Abbreviations:
hIAPP, human Islet Amyloid Polypeptide; rIAPP, rodent Islet Amyloid Polypeptide, CD, circular dichroism; LUVs, large unilamellar vesicles; NMR, nuclear magnetic resonance; T2DM, type-2 diabetes mellitus; TEM, transmission electron microscopy; ThT, Thioflavin T.
\end{abstract}




\begin{abstract}
Human islet amyloid polypeptide (hIAPP) is a highly amyloidogenic peptide found in pancreatic islets of type-2 diabetes (T2D) patients. Under certain conditions, hIAPP is able to form amyloid fibrils that play a role in the progression of T2D. hIAPP is synthesized in the $\beta$-cell of the pancreas and stored in the secretory granules before being released into the extracellular compartment. It has been suggested that natural stabilizing agents, such as insulin or zinc present in the secretory granules with hIAPP could prevent hIAPP fibril formation. The difference in the amino acid sequences of IAPP among species strongly correlates with amyloidogenicity and toxicity. The residue histidine at position 18 is known to be important in modulating the fibril formation, membrane leakage and toxicity. In this study, we have synthesized four analogues of hIAPP (H18R-IAPP, H18K-IAPP, H18A-IAPP and H18E-IAPP) and characterized their aggregation with either insulin or zinc in order to determine the effect of the residue-18 on the insulin-IAPP and zinc-IAPP interactions using a variety of biophysical experiments including thioflavin-T fluorescence, transmission electron microscopy imaging, circular dichroism, and NMR spectroscopy. We show that insulin reduced hIAPP fibril formation both in solution and in the presence of membrane and hIAPP-membrane damage and that the interactions are somewhat mediated by the residue-18. In addition, our results reveal that zinc affects the process of hIAPP fibril formation in solution but not in the presence of membrane. Our results indicate that the nature of the residue-18 is important for zinc binding. Based on this observation, we hypothesize that zinc binds to the residues in the N-terminal region of hIAPP, which is not accessible in the presence of membrane due to its strong interaction with lipids.
\end{abstract}

\title{
Keywords
}

Islet amyloid polypeptide; amylin; amyloid; type 2 diabetes mellitus; aggregation; membrane leakage.

\section{Highlight points}

- Importance of residue-18 in hIAPP self-assembly in the presence of insulin.

- Insulin slightly decreases the rate of hIAPP-membrane damage.

- Insulin slightly decreases hIAPP fibril formation in the membrane independent of the nature of residue-18.

- In solution, zinc enhances helical and anti-parallel $\beta$-sheet structures for H18R-IAPP and hIAPP, respectively.

- The presence of zinc does not modify IAPP induced membrane damage. 


\section{Introduction}

Diabetes is classified into two types: type-1 diabetes which is characterized by absolute insulin deficiency and type-2 diabetes (T2DM) that is defined by an inadequate insulin secretion (Butler et al. 2003). T2DM accounts for almost $95 \%$ of the diabetic patients and affects nearly 422 million people worldwide in (data WHO). TD2M thus belongs to the top 10 causes of death, reaching epidemic proportions and high health care costs. T2DM is characterized histopathologically by the presence of fibrillar amyloid deposits in the pancreas. These amyloid protein aggregates cause major cell dysfunctions and death, mainly in pancreatic $\beta$-cells but also in other organs, such as heart or kidney, contributing to the development and worsening of the diseases (Despa, 2012, Circ Res).

The major constituent of amyloid fibrils in T2DM is a 37 amino acid peptide, named islet amyloid polypeptide (IAPP) or amylin (Cooper et al. 1987, Westermark et al. 1987). IAPP is co-produced and cosecreted with insulin by $\beta$-cells in a molar ratio of about 1:100 in healthy individuals, but in a ratio of approximately 1:20 in diabetic patients since IAPP levels are elevated in conditions associated with insulin resistance (Hull et al. 2004, Enoki et al. 1992, Milardi et al. 2021). Under normal condition, IAPP plays an adaptive role in metabolism but in T2DM IAPP aggregates and is deposited as pancreatic islet amyloid plaques associated with reduced $\beta$ cell volume (Cooper et al. 1987, Clark et al. 1988, Hull et al. 2004, Westermark et al. 1987). A strong correlation was shown between the occurrence of T2DM in a particular species and the primary amino acid sequence, and the amyloid-forming propensity, of IAPP that is produced by that species. Earlier studies showed that the rodent-IAPP (rIAPP), that do not form fibrils, differ from human-IAPP (hIAPP) only at six positions out of 37, five of which are located between residues 20-29 (Betsholtz et al. 1994, Westermark et al. 1990). These observations led to the initial hypothesis that the amyloidogenic properties of IAPP is mainly dictated by the primary sequence in the 20-29 region. Nevertheless, it was realized later that this domain is not the only important segment of IAPP that drives the fibril formation. Indeed, a few studies showed that mutations within the N14, F15, L16, V17 and H18 residues induced a significant decrease of IAPP amyloid formation (Abedini et al. 2006, Brender et al. 2008, Tu et Raleigh 2013, Fox et al. 2013, Khemtemourian et al. 2017, Hoffman et al. 2018). Studies on hIAPP have shown that membranes play an active role in amyloid formation (Milardi et al. 2021, Rodriguez Camargo et al. 2017) and that the N-terminal domain (residues 1-19) is primarily responsible for the interaction of the hIAPP peptide with membranes (Caillon et al. 2014, Engel et al. 2006, Brender et al. 2008).

Both IAPP and insulin are produced as a prepropeptide and after cleavage by the prohormones convertase PC2 and PC3, mature IAPP and mature insulin are released in the secretory granules (Marzban et al. 2006, Paulsson et al. 2005, Marcinkiewicz et al. 1994). In $\beta$-cell granules, hIAPP has a concentration of 0.8-4.0 $\mathrm{mM}$ and do not form fibrils while in vitro hIAPP forms amyloid fibrils in a few hours even at very low concentrations (as low as $1 \mu \mathrm{M}$ ) (Knight et al. 2004). Therefore, it was thought that the composition of the $\beta$ cell granules containing many components could somehow affect hIAPP fibril formation and stabilize it in a nontoxic form. A few studies demonstrated that an acidic $\mathrm{pH}$ of around 5.5, mimicking the environment of the granules, protects hIAPP from fibril formation (Abedini et Raleigh, 2005, Brender et al. 2008, Khemtemourian et al. 2011, Jha et al. 2013, Brender et al. 2015). A previous study in the presence of membrane showed that i) the hIAPP precursors (ProhIAPP and ProhIAPP ${ }_{1-48}$ ) do not form fibrils and ii) both precursors reduced the level of hIAPP fibril formation from 100\% to around 60\% (Khemtemourian et al. 2009). In another study, it was observed that the flanking peptides issue from the maturation of ProhIAPP to hIAPP do not form fibrils but only slightly reduce hIAPP fibril formation (Salazar Vazquez et al. 2020). Another potential inhibitor present in a high concentration in the granules is insulin. Many studies have pointed out that insulin is the most potent inhibitor although the detailed mechanism of inhibition remains not clear. Insulin is able to bind not only to monomeric species but also to larger oligomeric hIAPP as it was shown that insulin inhibits hIAPP fibril formation at substoichiometric concentrations (Brender et al. 2011, Gilead et al. 2006, Cui et al. 2009, Westermark et al. 1996, Jaikaran et al. 2004, Janciauskiene et al. 1997, Larson et al. 2004, Baram et al. 2018, Nedumpully-Govindan et al. 2015). Others studies demonstrated that insulin and hIAPP form heterocomplexes that suppresses hIAPP fibril formation (Knight et al. 2008, Wei et al. 2009, Wiltzius et al. 2009). Another important component of granules is zinc $\left(\mathrm{Zn}^{2+}\right)$. Pancreatic $\beta$-cells have the highest levels of $\mathrm{Zn}^{2+}$ among all cell types, the zinc concentration could reach $10-20 \mathrm{mM}$ in the interior of the dense-core of granules (Formby et al. 1984; Foster et al. 1993). It is interesting to note that $\mathrm{Zn}^{2+}$ deficiency is a common symptom in T2DM and that mutations within the zinc transporter SLC30A8 of 
the secretory granules is also linked to an increase risk in T2DM, highlighting the interaction studies of the complex $\mathrm{Zn}^{2+} / \mathrm{hIAPP}$ (Taylor, 2005). The interaction of $\mathrm{Zn}^{2+}$ with hIAPP was indeed investigated by many groups to reveal the role of this metal ion on hIAPP aggregational properties (Atrián-Blasco et al. 2048, Bellia et al. 2014, Ge et al. 2017, Tian et al. 2019, Ilitchev et al. 2018). However, contrasting results concerning the possible inhibition, the affinity with $\mathrm{Zn}^{2+}$ and the bindings site have been reported. The first study showed that zinc has a dual effect on hIAPP fibril formation depending on the zinc concentration (Brender et al. 2010). Later two studies combining ITC and NMR experiments demonstrated that zinc did not bind to monomeric hIAPP but rather to oligomers and that zinc was able to stabilize these aggregates. It is reported that zinc mainly binds to the residue-18 (Salamekh et al. 2011; Brender et al. 2013). Recently, two molecular simulations studies suggested that zinc did not affect the structural features of hIAPP fibril formation but affects the initiation step of the self-assembly. In addition, studies revealed that zinc ion is able to bind not only the histidine residue but also the cysteines at positions 2 and 7 when the disulfide bridge is reduced (Wineman-Fisher et al. 2016; Wineman-Fishet et al. 2017). Finally, other studies indicated that histidine was not the only residue that could bind to zinc and that the $\mathrm{N}$-amino terminus part of hIAPP is an additional site of interaction (Erthal et al. 2016, Magri et al. 2020). Two amino-acids are directly involved in the $\mathrm{Zn}^{2+}$ binding, i.e., His 18 and Lys 1 . One difference between the sequence of hIAPP and the nonamyloidogenic and non-toxic rIAPP is the substitution of histidine- 18 of hIAPP with an arginine in rIAPP. A few other studies have reported the importance of the residue-18 in hIAPP fibril formation and hIAPPinduced cell toxicity by replacing histidine by arginine, lysine, alanine or glutamic acid (Khemtemourian et al. 2017, Hoffmann et al. 2018, Brender et al. 2008). Green and co-workers have synthesized mouse IAPP peptide where the arginine-18 has been replaced by histidine-18 (Green et al., 2003). This R18H-mIAPP is able to form fibrils pointing out the importance of this residue for hIAPP structure and fibril formation.

Therefore, within this research we wanted to determine the importance of histidine-18 in binding with insulin and zinc. We synthesized 4 mutated peptides of hIAPP by replacing histidine-18: with a basic amino acid, arginine (H18R) and lysine (H18K), with an acidic residue, the glutamic acid (H18E), and by a noncharged residue, alanine (H18A) (Fig. 1). We investigated the effects of insulin and $\mathrm{Zn}^{2+}$ on hIAPP aggregation in vitro in solution and in the presence of lipid membranes, since membranes influence the aggregational behavior of hIAPP (Caillon et al. 2014, Brender et al. 2012, Sciacca et al. 2012, Knight et al. 2004, Janson et al. 1999). We also examined the influence of insulin and $\mathrm{Zn}^{2+}$ in membrane barrier properties. Our results suggest that residue-18 is involved in both insulin and $\mathrm{Zn}^{2+}$ binding and that mutations within this residue lead to drastic changes in term of fibril formation but also in membrane damage.

\section{Material and methods}

\subsection{Materials}

1,2-dioleoyl-sn-glycero-3-phosphocholine (DOPC) and 1,2-dioleoyl-sn-glycero-3-phospho-L-serine (DOPS) were obtained from Avanti Polar Lipids (Alabaster, USA). Thioflavin T (ThT) and calcein were obtained from Sigma-Aldrich (Saint Quentin Fallavier, France).

\subsection{Sample preparation}

An essential criterion for measuring the fibril formation kinetics of amyloid peptides is to start with a monomeric form of the peptide. Therefore, peptide stock solutions were freshly prepared before all experiments using the same batch. Peptide solutions were prepared as described previously (Caillon et al. 2014; Brender et al 2008). Briefly, stock solutions were obtained by dissolving the peptide powder at a concentration of $1 \mathrm{mM}$ in hexafluoroisopropanol (HFIP) and by letting them incubate for an hour. Successively, HFIP was evaporated under a stream of dry $\mathrm{N}_{2}$ gas and further dried under vacuum in a desiccator for at least $30 \mathrm{~min}$. The resulting peptide film was dissolved at a concentration of $0.2 \mathrm{mM}$ in DMSO for the fluorescence experiments. For all experiments, we used the same DMSO $(2.5 \% \mathrm{v} / \mathrm{v})$ concentration and hence we were able to compare the experimentally measured parameters $\left(\mathrm{t}_{1 / 2}, \mathrm{k}\right.$ and the maximum of intensity).

\subsection{Preparation of phospholipid vesicles}

Lipid powders were carefully dissolved in chloroform. The solvent was evaporated under a stream of dry nitrogen gas in a Thermomixer ${ }^{\mathrm{TM}}\left(\sim 40^{\circ} \mathrm{C}\right)$ and further dried under high vacuum in a desiccator for at least $30 \mathrm{~min}$. Lipid films were then rehydrated at $37^{\circ} \mathrm{C}$ for $1 \mathrm{~h}$ with buffer $10 \mathrm{mM}$ Tris, $100 \mathrm{mM} \mathrm{NaCl}, \mathrm{pH} 7.4$, 
obtaining multilamellar vesicles (MLVs). After hydration, the vesicles were subjected to 10 freeze-thaw cycles using liquid $\mathrm{N}_{2}$ and a water bath $\left(\sim 40^{\circ} \mathrm{C}\right)$. Subsequently, large unilamellar vesicles (LUVs) were prepared by extruding the MLVs dispersion through an Avanti Mini-Extruder (Avanti Polar Lipids) equipped with a $200 \mathrm{~nm}$ Whatman polycarbonate filter, at least 19 times. Calcein-containing vesicles were obtained by adding $70 \mathrm{mM}$ calcein to the hydration buffer and free calcein was removed from the LUVs using size exclusion chromatography (Sephadex G50-fine), using the hydration buffer (10 mM Tris, $100 \mathrm{mM} \mathrm{NaCl}$, $\mathrm{pH}$ 7.4) to as the mobile phase. Calcein stock solution was dissolved in $300 \mu \mathrm{L}$ tris $500 \mathrm{mM}, 300 \mu \mathrm{L} \mathrm{NaOH}$ $2 \mathrm{M}$ in water to obtain a final concentration of $50 \mathrm{mM}$ tris. The calcein stock solution contained $300 \mu \mathrm{L}$ $\mathrm{NaOH}$ at $2 \mathrm{M}$, thus the salt concentration was identical inside and outside the lipid vesicles. Phospholipid concentrations of LUVs were determined by a phosphate assay (Rousser et al. 1970).

\subsection{Fluorescence assays}

The effect of insulin and zinc $\left(\mathrm{ZnCl}_{2}\right)$ on the kinetics of IAPP fibril formation was measured both in solution and in the presence of lipid membranes (LUVs) by following the fluorescence intensity increase upon Thioflavin T (ThT) binding to fibrils. A CLARIOstar ${ }^{\circledR}$ Plus spectrophotometer (BMG LabTech, Germany) and a black 96-well microtiter plate reader (Greiner CELLSTAR ${ }^{\circledR}$ 96-well black microtiter plate polystyrene flat-bottom wells) were used. ThT fluorescence was measured at room temperature every 10 min with $450 \mathrm{~nm}$ excitation filter and $483 \mathrm{~nm}$ emission filter. Fluorescence assays were started by adding $5 \mu \mathrm{L}$ of a $0.2 \mathrm{mM}$ IAPP $(5 \mu \mathrm{M}$ peptide) to $195 \mu \mathrm{L}$ of a mixture of $100 \mu \mathrm{M}$ ThT, DOPC/DOPS (7:3) LUVs, with a peptide:lipid ratio of 1:20 (for experiments in the presence of membranes), buffer $10 \mathrm{mM}$ Tris, $100 \mathrm{mM}$ $\mathrm{NaCl}, \mathrm{pH} 7.4$, insulin or $\mathrm{ZnCl}_{2}$, at 4 different peptide:insulin ratio (1:1, 1:5, 1:10, and 1:20) and peptide:zinc ratio $(1: 5,1: 20,1: 100$, and 1:1000). After the addition of all the components, an initial orbital shake (10 s, $500 \mathrm{rpm}$ ) was performed. The assays were performed in triplicate. The results presented here are the average of 3 different measurements fitted by applying the Boltzmann sigmoidal equation.

\subsection{Membrane leakage assays}

The influence of insulin and zinc on the permeability of membranes to IAPP fibrils was measured by following the increasing of calcein fluorescence due to leakages in calcein-loaded DOPC/DOPS (7:3) LUVs. A CLARIOstar ${ }^{\circledR}$ Plus spectrophotometer (BMG LabTech, Germany) and a clear 96-well microtiter plate reader (Corning 96-well clear flat bottom polystyrene not treated microplate) were used. Calcein fluorescence was measured at room temperature every 10 min with $483 \mathrm{~nm}$ excitation filter and $530 \mathrm{~nm}$ emission filter. The peptides at $5 \mu \mathrm{M}$ were added to a mixture of calcein-containing LUVs in buffer $10 \mathrm{mM}$ Tris, $100 \mathrm{mM} \mathrm{NaCl}, \mathrm{pH} 7.4$ (peptide:lipid ratio of 1:20), at 4 different peptide:insulin ratios (1:1, 1:5, 1:10, and 1:20) and at 3 different peptide:zinc ratios (1:5, 1:20, and 1:100). Directly after the addition of all peptides, the microtiter plate was shaken for $10 \mathrm{~s}$ at $500 \mathrm{rpm}$ before starting the fluorescence detection. The fluorescence intensity was measured at room temperature. The maximum value of leakage-induced fluorescence in each well was determined upon the addition of $2 \mu \mathrm{L}$ of $10 \%$ Triton X-100. The release of fluorescent dye was normalized according to the following equation:

$$
L(t)=\frac{\left(F_{t}-F_{0}\right)}{\left(F_{100}-F_{0}\right)}
$$

where $\mathrm{L}(\mathrm{t})$ is normalized fluorescence intensity of the dye released (due to membrane leakage), $\mathrm{F}_{\mathrm{t}}$ is the measured fluorescence intensity, and $\mathrm{F}_{0}$ and $\mathrm{F}_{100}$ are the fluorescence intensities at times $\mathrm{t}=0$ and after addition of Triton X-100, respectively. The calcein dye-leakage experiment was performed in triplicate. The results presented here are the average of 3 different measurements fitted by applying the Boltzmann sigmoidal equation.

\subsection{Electron microscopy}

Aliquots $(20 \mu \mathrm{L})$ of the samples used for fluorescence assays were removed at the end of each kinetic experiment, blotted on a carbon coated 200 mesh copper grids, glow-discharged for $45 \mathrm{~s}$, and then negatively stained with 4\% uranyl acetate for 1 min. The grids were dried and examined using a FEI-CM 120 transmission electron microscope equipped with a US1900 GATAN CCD camera. 


\subsection{Statistical analysis}

Data of the fluorescence assays were analyzed by non-linear regression to fit the theoretical Boltzmann sigmoidal with the experimental outcome using PRISM (Graphpad Software Inc.) according the following equation:

$y(x)=\frac{y_{\min }+\left(y_{\max }-y_{\min }\right)}{1+e^{\frac{t_{1 / 2}-x}{\alpha}}}$

Fluorescence intensity values were plotted using an asymmetrical confidential intervals level of the parameters of $95 \%$ and prediction bands based on the asymmetrical profile likelihood. The maximum of intensity, the lag-time $\mathrm{t}_{1 / 2}$, and elongation rate $(\mathrm{k})$ values were extrapolated from the Boltzmann sigmoidal best-fit values.

\subsection{NMR experiments}

NMR measurements were done to investigate the effect of zinc on two of the IAPP peptides, wild-type hIAPP and H18R-IAPP. HFIP treated and lyophilized peptide (wild-type and H18R-IAPP) samples were dissolved in $10 \mathrm{mM}$ Tris-d11, $100 \mathrm{mM} \mathrm{NaCl}, \mathrm{pH} 7.4$ buffer to a final concentration of $100 \mu \mathrm{M}$ on ice. NMR samples (each with a total volume $\sim 600 \mu \mathrm{L}$ ) containing $65 \mu \mathrm{M}$ peptide, $10 \% \mathrm{D}_{2} \mathrm{O}$ mixed with/without 5 molar excess of $\mathrm{ZnCl}_{2}$ were prepared using the same peptide stock solution. NMR measurements were first done on the IAPP sample (hIAPP and H18R-IAPP) right after ( 15 minutes) the sample preparation following which zinc-containing sample (hIAPP/H18R-IAPP $+\mathrm{ZnCl}_{2}$ ) was prepared for NMR measurements. While time-lapse NMR experiments were carried out on the IAPP sample without zinc, the zinc-containing peptide sample solution was flash-frozen to avoid early peptide aggregation. The flash-frozen stock IAPP sample was thawed on ice followed by a short vortex and $\sim 30 \mathrm{~s}$ sonication prior to the preparation of IAPP $+\mathrm{ZnCl}_{2}$ NMR sample. Proton NMR spectra were recorded using 512 scans and a 2 s recycle delay with a total acquisition time of $\sim 30 \mathrm{~min}$. Then, diffusion ordered NMR spectroscopy (DOSY) experiments were carried out using stimulated-echo with bipolar gradient pulses for diffusion with a 2 to $98 \%$ gradient strength increment. DOSY spectra were acquired with 16 gradient strength increments, 36,000 time-domain data points in the $t_{2}$ period, $2 \mathrm{~s}$ recycle delay, and a $200 \mathrm{~ms}$ diffusion delay. All NMR samples after measurements were incubated at room temperature and proton NMR spectra were recorded at $\sim 22 \mathrm{~h}$ and $\sim 96 \mathrm{~h}$ to monitor peptide aggregation. All NMR measurements were done on a $500 \mathrm{MHz}$ Bruker NMR spectrometer at $25{ }^{\circ} \mathrm{C}$ equipped with a TXI $\left({ }^{1} \mathrm{H} /{ }^{13} \mathrm{C} /{ }^{15} \mathrm{~N}\right)$ probe. All NMR spectra were processed using Bruker Topspin 3.5 and DOSY analysis was carried out using Topspin Dynamic Center. Integral of 1D proton NMR signals (in the 6.5 to $8.7 \mathrm{ppm}$ region) were calculated using MestReNova 12.0.4.

\subsection{Circular Dichroism experiments}

NMR samples post $\sim 96 \mathrm{~h}$ measurement were gently mixed by vortexing for $\sim 30 \mathrm{~s}$, and then transferred to a $1.5 \mathrm{~mL}$ centrifuge tube. About $200 \mu \mathrm{L}$ of the NMR sample was transferred to a $1 \mathrm{~mm}$ path length quartz CD cuvette. CD measurements were carried out on all four NMR samples at $25^{\circ} \mathrm{C}$, and data were averaged from 6 scans after subtracting the buffer (baseline) spectrum. The CD spectra were analyzed using the BestSel program (Micsonai et al., 2015) to quantify the secondary structure contents in each of the samples.

\begin{tabular}{|c|c|}
\hline IAPP & 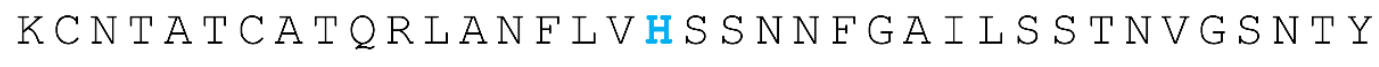 \\
\hline rIAPP & R L A N F L V R S S N N L G P V L P P T N V G \\
\hline & R L A N F L V R S S N N F \\
\hline & A N F L V F \\
\hline & $N E L V E$ \\
\hline & $\mathrm{T} T T_{\mathrm{z}} \mathrm{C}$ \\
\hline
\end{tabular}

Fig. 1. The primary amino acid sequence of native human-IAPP (hIAPP), the rodent-IAPP (rIAPP) and the mutated peptides investigated in this study. The mutation of the residue-18 in hIAPP (highlighted in blue) is 
highlighted in bold (dark blue for rodent IAPP, H18R, purple; H18K, red; H18E, green; and H18A, yellow). Each of the peptide contains a disulfide bond between Cys-2 and Cys-7 and an amidated C-terminus.

\section{Results and discussion}

\subsection{Importance of residue 18 in hIAPP self-assembly in the presence of insulin}

In the previous studies, we showed the importance of residue-18 on the self-assembly and aggregation kinetics of hIAPP (Khemtemourian et al. 2017, Hoffmann et al. 2018, Brender et al. 2008,). Here we have determined the full kinetics of the wild-type hIAPP and its mutant peptides, in the absence and in the presence of insulin, to assess the importance of the histidine-18 residue on the insulin binding. hIAPP and the mutant peptides were incubated with insulin at different molar ratios, $1: 1 ; 1: 5 ; 1: 10$ and 1:20, to mimic the environment of granules. Fig. 2a-e shows typical sigmoidal curves obtained for wild-type and the mutated hIAPP without and with insulin. As observed previously, wild-type hIAPP forms amyloid fibrils more rapidly than the mutant peptides (Khemtemourian et al. 2017). A significant difference in the trend between the peptide alone and the insulin-treated peptides were observed independently from the nature of the residue-18. Indeed, the addition of insulin to all the peptides slowed down the kinetics of fibril formation. The kinetics data were fitted according to a Boltzmann sigmoidal equation. Three parameters were derived from the ThT curves: $t_{1 / 2}$, the time a half fibril conversion, the elongation rate and the fluorescence intensity at the plateau (Fig. 2f-j). Fig. 2f,2k show that the lag-time $\left(t_{1 / 2}\right)$ and the elongation rate $(k)$ of the wild-type IAPP increased in a monotonic and nearly linear fashion with the varying concentrations of insulin. The $\mathrm{t}_{1 / 2}$ is at $3.8 \mathrm{~h}$ for hIAPP alone and reached around $19 \mathrm{~h}$, a 5 -fold longer, in the presence of insulin at a molar ratio of 1:20. The addition of insulin to H18R- and H18K-IAPP enhanced the lag time, respectively by a 3fold and a 2-fold, independent of insulin concentration, suggesting a different mode of interaction (Fig. $2 \mathrm{~g}, 2 \mathrm{~h}$ ). Insulin also slows down the kinetics of fibril formation for H18A- and H18E-hIAPP (Fig 2i,2j). For those four peptides, the most inhibition is obtained with the molar ratio of 1:1, suggesting that insulin binds to the monomeric form of the peptides. The observed ThT fluorescence results indicate that insulin interacts with all hIAPP peptides in a different manner and this interaction induces a significant decrease in the fibril formation. The maximum of ThT is always higher in the presence of insulin for all of the peptides suggesting that insulin-hIAPP binding might interfere with ThT dye binding to the fibers (Fig 2f,2j). Thus, in order to investigate the effect of insulin on the morphology of the insoluble aggregates, aliquots were removed at the end of each kinetic run (at a molar ratio 1:20 of IAPP:insulin), dried on the TEM grids, and imaged with transmission electron microscopy. In the absence of insulin, hIAPP fibrils exhibit the typical dense mats of the long branched fibrils, characteristic of amyloid proteins (Fig 2k) in agreement with previous studies (Khemtemourian et al. 2011, Brender et al 2011, Ke et al. 2017). The addition of insulin caused a significant change in the morphology and in the amounts of deposits. Small amorphous aggregates were observed in large quantities accompanied with thin and short fibrils (Fig 2p). The same results were obtained for all the mutated peptides, i.e., insulin considerably affected the morphology of the fibrils. Amorphous aggregates from different sizes were found for H18R-, H18E- and H18A-IAPP, and short and thin fibrils were detected for H18K-IAPP (Fig.2 q,t). Altogether our results 

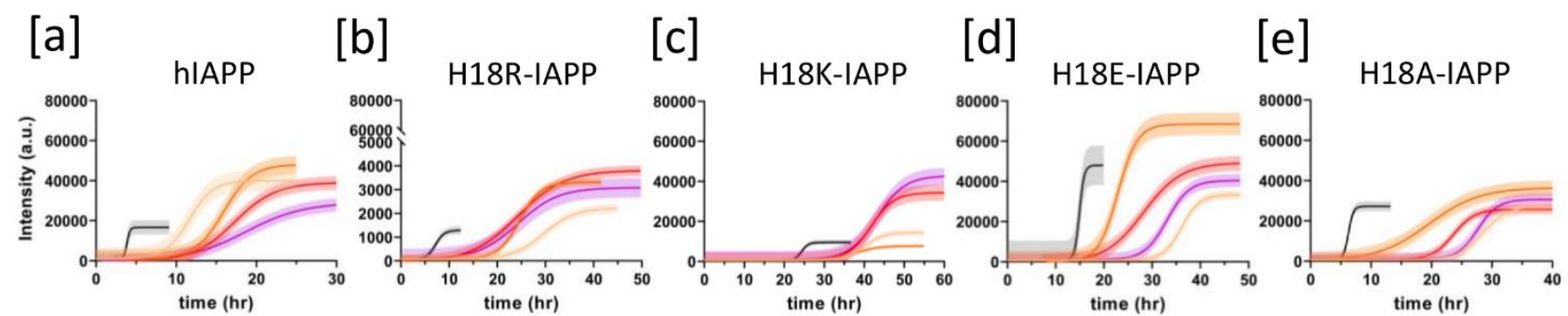

[f]
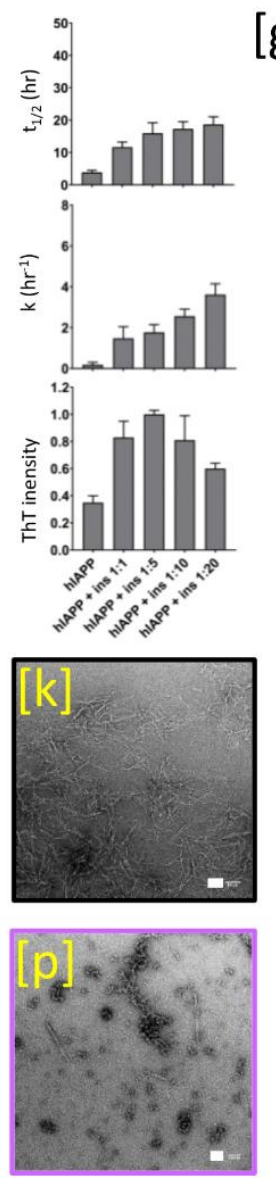
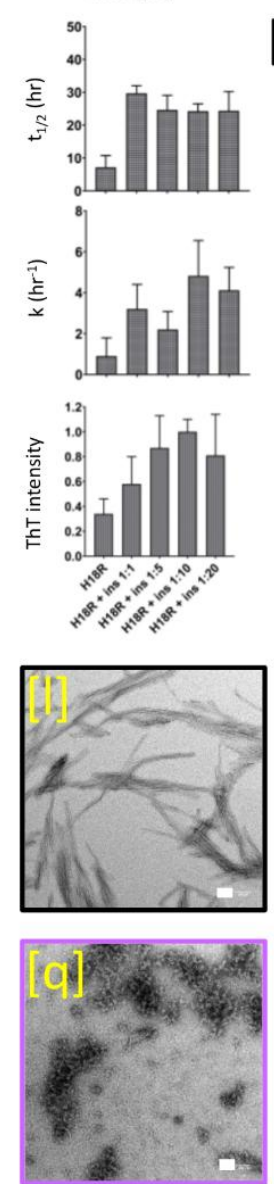

[h]
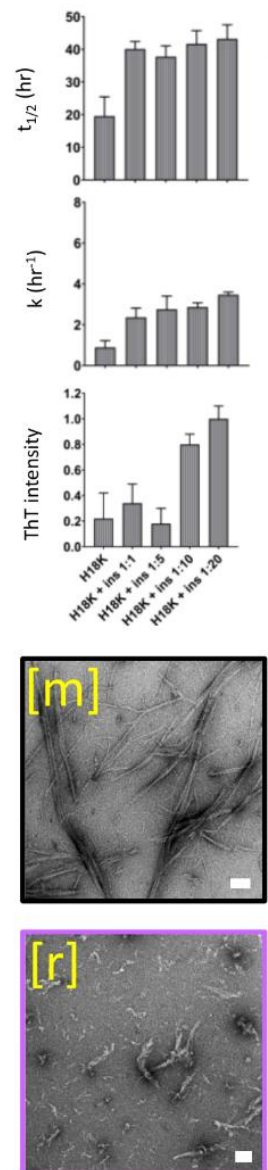

[i]
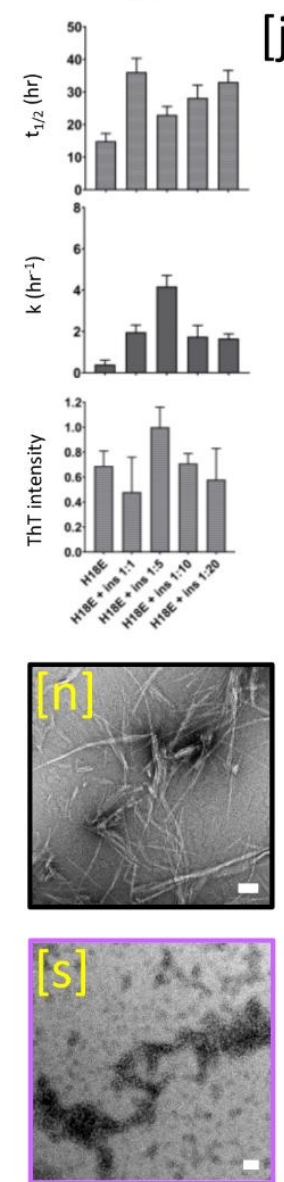

[j]
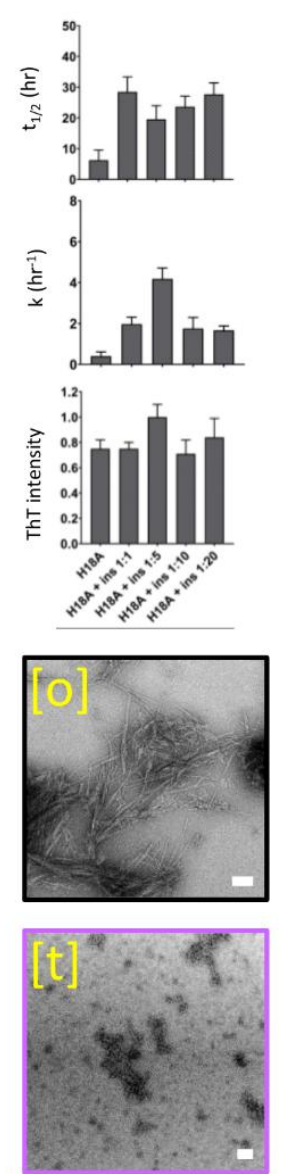

Fig. 2. Effect of insulin on the kinetics of fibril formation in solution. Representative fit curves for wild-type hIAPP (a, black) and its mutant peptides alone (b-e, black) and in the presence of insulin with molar ratio of 1:1 (yellow), 1:5 (orange), 1:10 (red) and 1:20 (purple). The kinetics parameters, i.e. $\mathrm{t}_{1 / 2}$, elongation rate and maximum intensity are indicated in the bar charts ( $\mathrm{f}-\mathrm{j})$. The lag-time and elongation rate were obtained by fitting the data to a Boltzmann sigmoidal model and prediction bands are plotted with a confidence level of 95\%. Maximal ThT fluorescence intensities were determined once the plateau phase was reached. The error bars shown (f-j) were calculated as SEM of three independent measurements in triplicate. TEM images of hIAPP and the mutated peptides alone (k-o) and in the presence of insulin (molar ratio of 1:20, p-t) after 3 days of kinetic measurements at $5 \mu \mathrm{M}$ in solution are shown with scale bars indicating $100 \mathrm{~nm}$.

indicated that insulin obviously inhibits mature hIAPP fibril formation in a dose-dependent manner as described previously (Wang et al., 2014; Brender et al 2011), while insulin reduces the fibril formation of hIAPP-mutants to a less extent, suggesting the importance of residue-18 in the insulin-IAPP interaction. It was indeed shown that the major binding between insulin and hIAPP was thought that the chain-B of insulin and the residues 8-18 of hIAPP (Susa et al., 2014; Gilead et al., 2006). In addition, an NMR study demonstrated that the salt bridge between Arg-11 and His-18/Arg-18 of hIAPP/rIAPP and Glu-13 of insulin chain-B triggers the IAPP-insulin interactions (Wei et al., 2009) suggesting that the interaction might be 
stabilized primarily by positively charged residues of IAPP. However, our experiments at a 1:1 molar ratio indicated that insulin is able to inhibit the aggregation of all of the mutant peptides, suggesting that the residue-18 is not the only residue that mediates IAPP-insulin interactions. The global charge of insulin is neutral, but the chain A contains 2 negatively charge amino acids while the chain B is positively charged. Our results suggest that since insulin is able to bind to all the mutated peptides independently of the charge, the domain of hIAPP interacting with insulin might somehow change in function of the residue 18 . We speculate that hIAPP and the H18R-IAPP and H18K-IAPP will bind to the chain B while the H18E-IAPP will bind to the chain A. It is interesting to note that for all the peptides the fibril morphology in the presence of insulin differs from that observed in the absence of insulin. This result indicates that insulin affects IAPP (wild-type and mutants) assembly as it was previously shown for wild-type hIAPP (Larson et Miranker, 2004; Brender et al. 2011).

\subsection{In the membrane, insulin slightly decreases the rate of hIAPP-membrane damage and hIAPP fibril formation independent of the nature of residue-18}
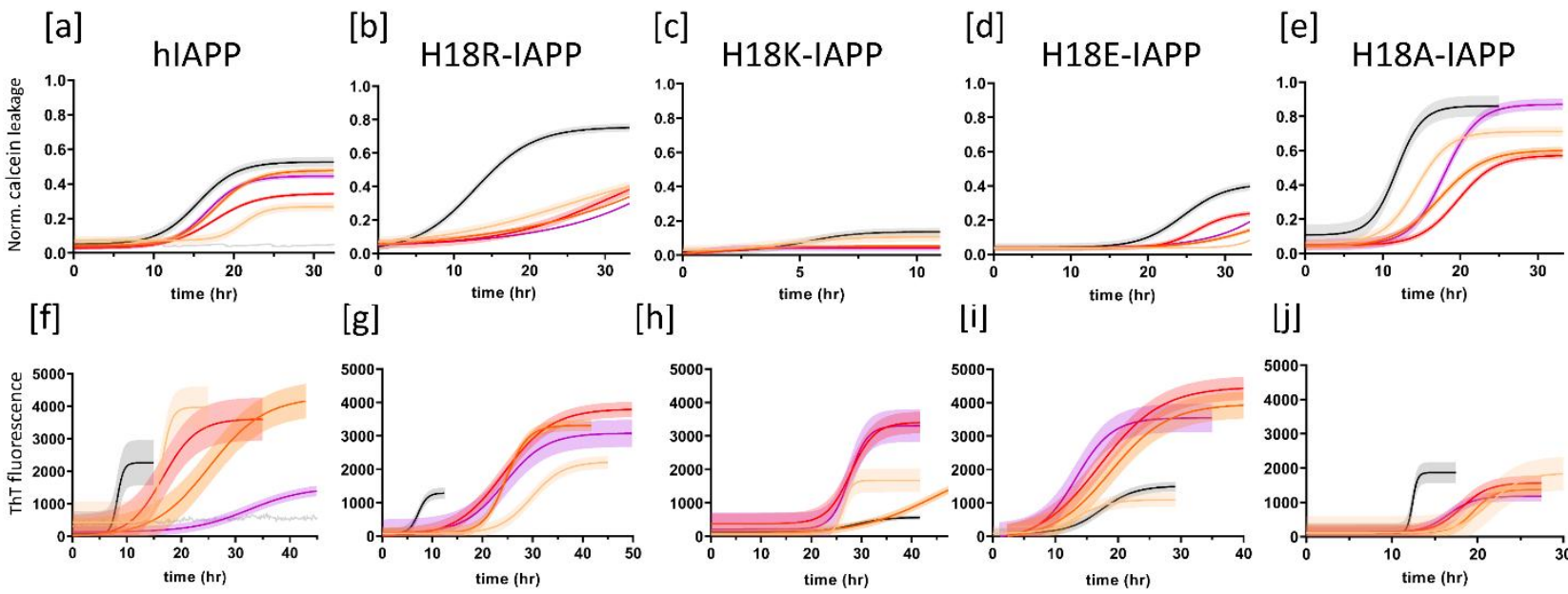

[h]
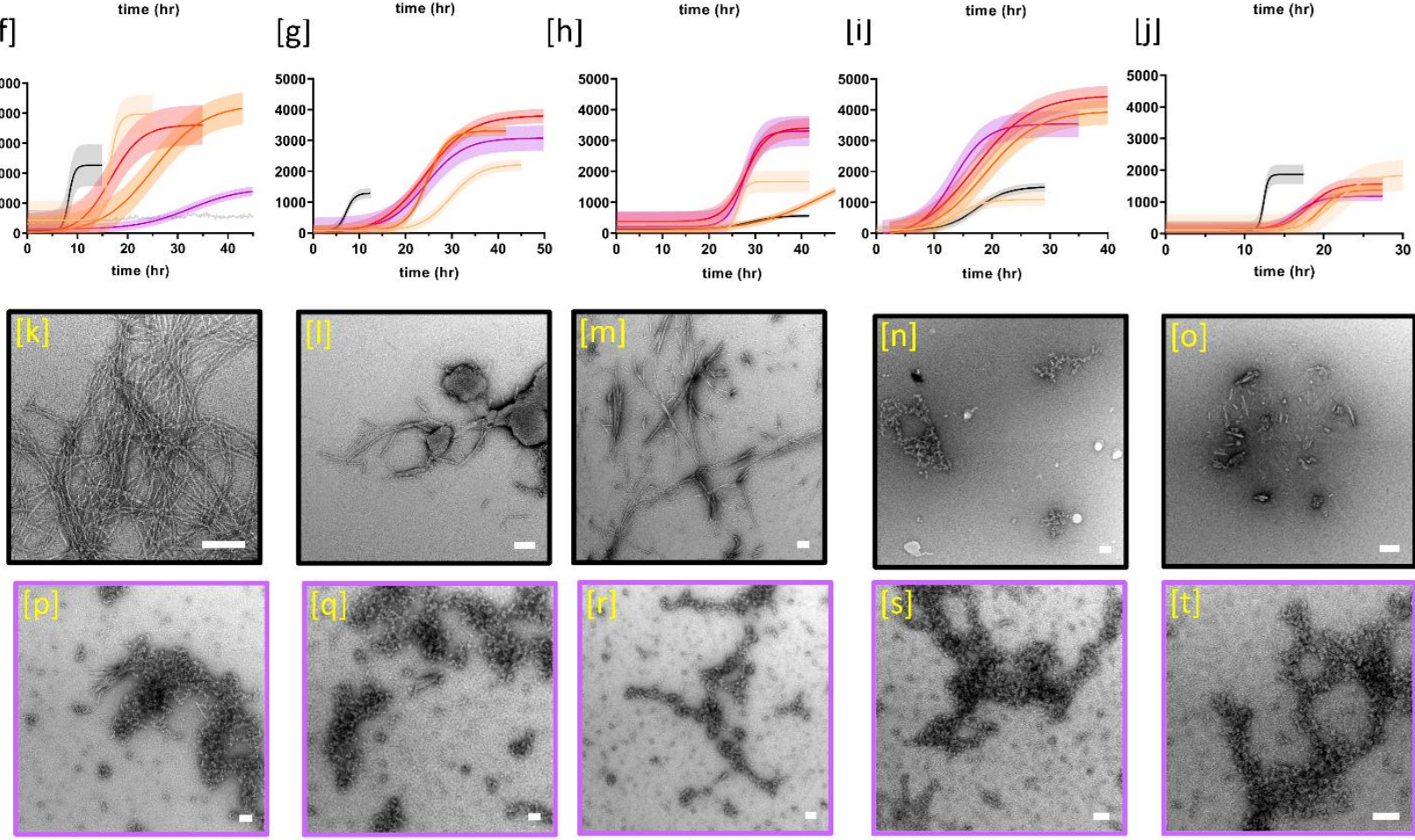

Fig. 3. Effect of insulin on IAPP-induced membrane disruption. Inhibition of IAPP-induced membrane damage in the presence of insulin (a-e) at a peptide:lipid ratio of 1:20. Curve fitting of fluorescence intensity data from calcein leakage induced by wild-type hIAPP (a, black) and the mutant peptides (b-e, black) in the presence of insulin with a molar ratio of: 1:1 (yellow), 1:5 (orange), 1:10 (red) and 1:20 (purple). Inhibition of IAPP fibril formation by insulin in a lipid bilayer (f-j). ThT fluorescence intensity of hIAPP (f) and the mutant peptides ( $\mathrm{g}-\mathrm{j}$ ) with increasing insulin:hIAPP molar ratios: 1:1 (yellow), 1:5 (orange), 1:10 (red) and 1:20 (purple). The signal of zinc alone is given in a and f(light grey). Negative stain TEM images of hIAPP and the mutant peptide alone (k-o) and of the aggregates formed after incubation with 1:20 molar ratio insulin (p-t). Scale bars in the TEM images (k-t) indicating $100 \mathrm{~nm}$.

Although the mechanism of membrane disruption induced by hIAPP has not been fully elucidated, the propensity of hIAPP to interact with and damage the membrane is well established (Mirzabecov et al. 1996, Quist et al. 2005, Anguiano et al. 2002, Porat et al. 2003, Kayed et al. 2004, Janson et al. 1999, Engel et al. 
2008, Sciacca et al 2012, Brender et al. 2007, Brender et al. 2013). To assess the influence of insulin on hIAPP-induced membrane damage, we measured the calcein leakage induced by hIAPP with and without insulin from DOPC/DOPS vesicles (7:3). The damage induced by prefibrillar species of hIAPP was evaluated by measuring calcein dye released directly after the addition of various concentration of insulin (from $5 \mu \mathrm{M}$ to $100 \mu \mathrm{M}$ ) of freshly dissolved hIAPP to $100 \mathrm{mM}$ of 7:3 DOPC/DOPS liposomes. A time-trace of calcein leakage induced by $5 \mu \mathrm{M}$ of wild-type or mutated hIAPP in the absence and in the presence of insulin is shown in Fig.3A-E. Our data for the wild-type hIAPP indicates that the presence of insulin decreases the extent of leakage from $55 \pm 5 \%$ for hIAPP alone to $25 \pm 5 \%$ for hIAPP in the presence of insulin (hIAPP:insulin, 1:1) of total vesicles content measured after $30 \mathrm{~h}$. Only small differences in lag-time were observed, $\mathrm{t}_{1 / 2}$ varied from 15 to $21 \mathrm{hr}$ without and with insulin, respectively. For all mutated peptides, insulin also reduces the maximum of leakage and increases the lag-time, suggesting that in the presence of membranes, insulin interacts with hIAPP independently of the mutations. For H18R-IAPP, our data show that the presence of insulin inhibits notably membrane leakage induced by the peptides, by reducing the lagtime from around $14 \mathrm{hr}$ to more than $35 \mathrm{hr}$. For H18K-IAPP, H18E-IAPP and H18A-IAPP, insulin reduces both the maximum extent of leakage and the lag-time, suggesting that the charge of the residue- 18 does not influence insulin binding in the presence of membranes. The next step was to test the effect of insulin on hIAPP fibrillation in the presence of membrane vesicles. As in the solution experiments, the aggregation kinetics of hIAPP and the mutated peptides were studied by a ThT binding assay, but now in the presence of DOPC/DOPS LUVs (7/3). In the presence of membranes, insulin has also an important influence on mature hIAPP fibril formation. The $t_{1 / 2}$ is almost 5-fold longer in the presence of insulin (1:20 molar ratio) than in the absence of insulin. For the peptides H18R-IAPP and H18A-IAPP, insulin seems to reduce fibril formation but to a less extent than for the wild-type. In contrast, insulin accelerates the rate of fibril formation of both H18K-IAPP and H18E-IAPP. The maximum of intensity of the peptide in the presence of insulin is higher or as high as the one of the peptides alone, suggesting that the presence of insulin might change the fibril morphology. Therefore, the samples from the ThT experiments were stained, blotted and observed by TEM. Fig. 3k,o shows the images of the peptides without insulin, and Fig.3p,t display the images of the peptide with insulin. In the absence of insulin after 5 days of incubation, typical long and twisted fibrils were observed for the wild-type hIAPP while for H18K-IAPP and H18R-IAPP only thin and small fibrils were observed and only amorphous aggregates were detected for H18A-IAPP and H18E-IAPP, consistent with a previous report (Hofmann et al. 2018). The addition of insulin lead to some changes in the morphology of the aggregates. For all the peptides, including the wild-type hIAPP, amorphous aggregates were detected, however no fibrils were identified even after 5 days of incubation. In the presence of membrane, insulin inhibits hIAPP fibril formation and membrane damage independent of the mutation indicating that the residue-18 is not the only residue involved in the insulin interaction. It was shown that the hetero-complex hIAPP-insulin is able to interact with the lipid membrane, albeit to a lesser extent than hIAPP alone (Sellin et al. 2010). Since insulin has a strong affinity with membrane, we assume that insulin somehow inhibits the fibril formation less for hIAPP and the mutated peptides in the presence of membranes than in solution. Insulin weakened the hIAPP induced membrane damage is probably due to the formation of the hetero-complex hIAPP-insulin in the bulk aqueous phase preventing membrane interaction and thus membrane leakage. In the presence of membrane, insulin also alters the fibril morphology for all the peptides as in the absence of membranes.

\subsection{Effect of zinc binding on the fibril formation kinetics of IAPP}

Zinc is present in a high concentration in the secretory granules. According to in vitro findings, zinc can either enhance or inhibit hIAPP aggregation depending on the hIAPP:zinc ratio (Westermark at al. 1996; Salamekh et al. 2011; Brender et al. 2010). To determine if zinc could affect hIAPP amyloidogenesis and if this effect is due to the residue-18, we monitored the rate of fibril formation of $5 \mu \mathrm{M}$ of wild-type hIAPP and the mutated IAPP in the presence of 25, 100, 500 and $5000 \mu \mathrm{M} \mathrm{ZnCl}_{2}$. At a low hIAPP:zinc ratio (i.e, 1:1, 1:5 and 1:20), zinc significantly affects the formation of IAPP amyloid fibrils, as shown by the increase of $\mathrm{t}_{1 / 2}$ and the decrease of the intensity, in agreement with a previous report (Brender et al. 2010) (Fig. 4a,e). At a higher hIAPP:zinc ratio (1:100 and 1:1000), zinc has a moderate effect on hIAPP fibril formation. Different behaviors were observed for the mutated peptides. Zinc accelerates H18K-IAPP fibril formation at all the ratios. However, for H18A-IAPP, zinc does not have any effect on the fibril formation, the value of $t_{1 / 2}$ and of the maximum of intensity are not changed in the presence of zinc. Zinc has a dual effect on H18EIAPP and H18R-IAPP. At low concentrations, zinc seems to increase the $t_{1 / 2}$ and at high concentrations, zinc 
decrease the maximum of intensity. Our results demonstrated that zinc induces more variation on the fibril formation by the wild-type hIAPP, suggesting that zinc mainly bind to the imidazole of the histidine residue and to a less extend to other residues through a nonspecific binding.

We confirmed the results of the ThT assay by examining the structure of the amyloid fibrils using TEM. In the absence of insulin, TEM images of wild-type hIAPP and mutated peptides present the same
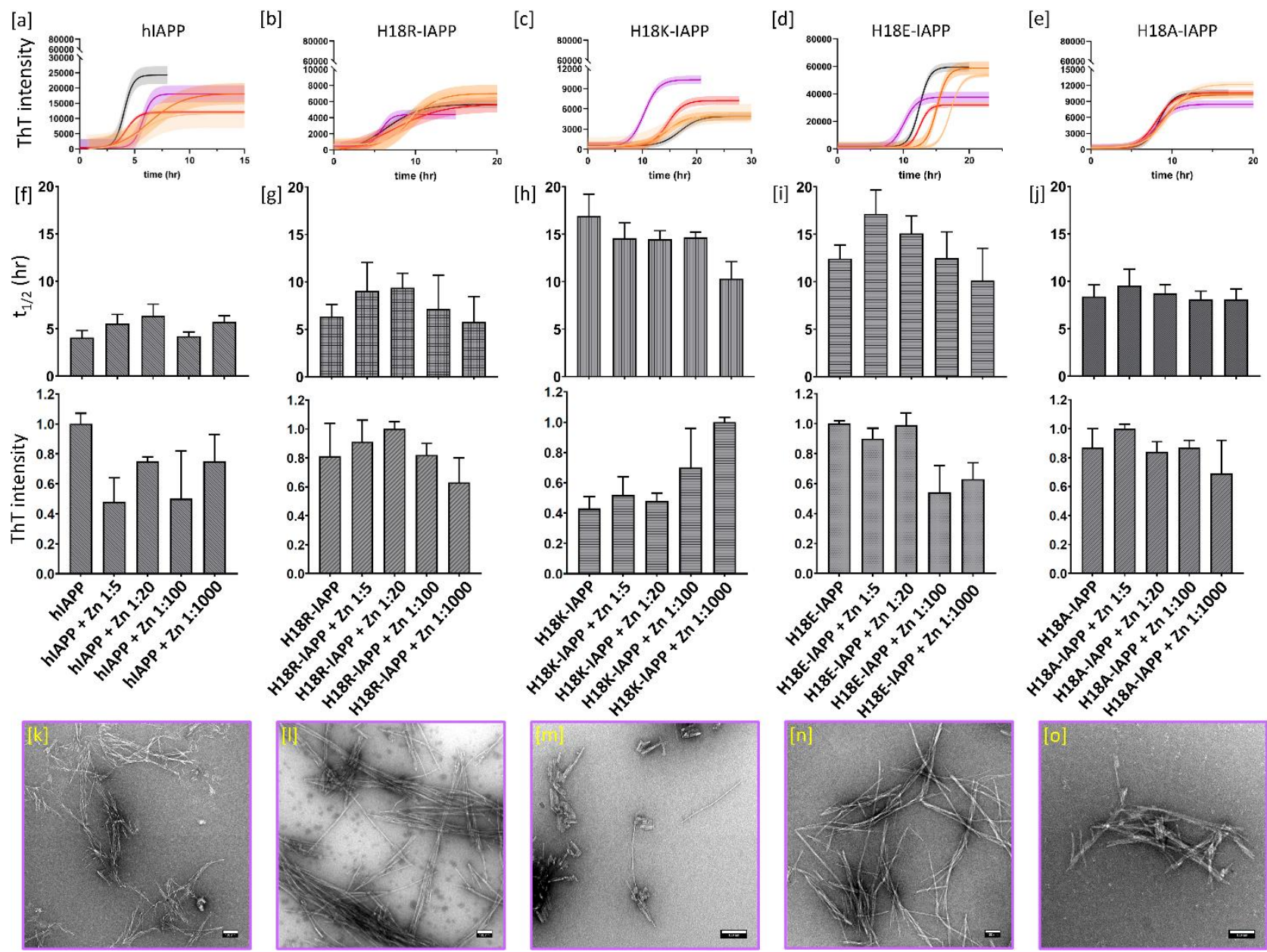

morphology as given in Fig.2k,o. In the presence of $1000 \mu \mathrm{M}$ zinc, the overall morphology of the fibrils remains similar for wild-type IAPP, H18R-IAPP and H18E-IAPP. Shorter and thinner fibril are observed for the peptides H18K-IAPP and H18A-IAPP. Amorphous aggregates or aggregates with aberrant morphology are not observed in the presence of zinc for wild-type IAPP neither for the mutated peptides.

Fig. 4. Effect of zinc on the kinetics of fibril formation in solution. Representative fit curves for wild-type hIAPP (a, black) and the mutant peptides alone (b-e, black) and in the presence of zinc with molar ratio 1:5 (yellow), 1:20 (orange), 1:100 (red) and 1:1000 (purple). $\mathrm{t}_{1 / 2}$ and the maximum of intensity are shown for native and mutated hIAPP (fj). Electron micrographs showing the effect of zinc on the fibril morphology for wild-type and mutated hIAPP at a ratio 1:1000 (k-o). Scale bars in the TEM images (k-o) indicating $100 \mathrm{~nm}$.

It was shown that zinc binds tightly to the residue His 18 but an additional residue could likely act as a second ligand. A few studies proposed that the N-terminal part of the Lys1, the Ser19 or the His18 of another hIAPP could also bind to zinc (Magri et al. 2020, Brender et al. 2010). Our results show that zinc does not modify IAPP fibril formation for H18A-IAPP suggesting that the nature of the residue-18 is important for the zinc binding; since fibril morphology may not be sensitive to probe the zinc binding sites, additional studies utilizing high-resolution structures could be valuable although the presence of heterogeneous species of IAPP could pose numerous challenges. While zinc seems to slow down the kinetics of hIAPP fibril formation, the final fibrils formed are similar to those formed in the absence of zinc, in contrast to insulin which reduced the amount of fibrils and induced a change in fibril morphology as previously observed (Salamekh et al. 2011). 


\subsection{Zinc enhances helical and anti-parallel beta-sheet structures for H18R-IAPP and hIAPP, respectively, in solution}

As evidenced from the fluorescence assay, low concentration of zinc affects hIAPP fibrillation. To better understand how zinc binding at low concentration is influenced by the mutation of residue-18 in IAPP at an atomic level, we performed NMR and CD experiments on zinc-containing samples with 1:5 peptide:zinc molar ratio. We selectively compared the zinc binding to wild-type hIAPP and H18R-IAPP that showed a difference in the aggregation kinetics and fibril mass (Figure 4a and 4b). Proton NMR spectra showed zinc binding to hIAPP affect the chemical shift and signal intensities of histidine imidazole protons $\varepsilon 1$ and $\delta 2$ (Figure 5a) within $\sim 30$ minutes. In addition, the zinc binding presented an overall decrease in amide and aromatic proton signal intensities (Figure $5 \mathrm{~b}$ ). On the contrary, zinc binding to H18R-IAPP presented a slight increase in NMR signal intensity and did not show a change in the chemical shift values for most of the amide/aromatic proton signals (Figure 5a and b). The observed decrease and increase in proton NMR signal intensities of hIAPP and H18R-IAPP samples, respectively, can be correlated to a variation in the molecular size that could affect the molecular tumbling rate and the transverse relaxation rate $\left(\mathrm{R}_{2}\right)$; and/or due to the contribution from the conformational exchange that has been previously demonstrated for $\beta$-amyloid peptide (Sahoo et al., 2019; Yamaguchi et al., 2011). To investigate whether or not the observed line-broadening in hIAPP- $\mathrm{ZnCl}_{2}$ is size dependent, we measured the diffusion constants for hIAPP and H18R-IAPP in the absence and presence of $\mathrm{ZnCl}_{2}$. DOSY NMR analysis as shown in Figure $5 \mathrm{c}$ showed a diffusion rate constant (D) in the order of E- $09 \mathrm{~m}^{2} / \mathrm{s}$ for hIAPP; whereas zinc binding to hIAPP is shown to slightly increase the size with a measured diffusion constant of order E-10 $\mathrm{m}^{2} / \mathrm{s}$ (Table 1). On the other hand, a very small change in the diffusion coefficient values (in the order of E-10 m²/s) was observed for H18R-IAPP in the absence and presence of zinc (Table 1).
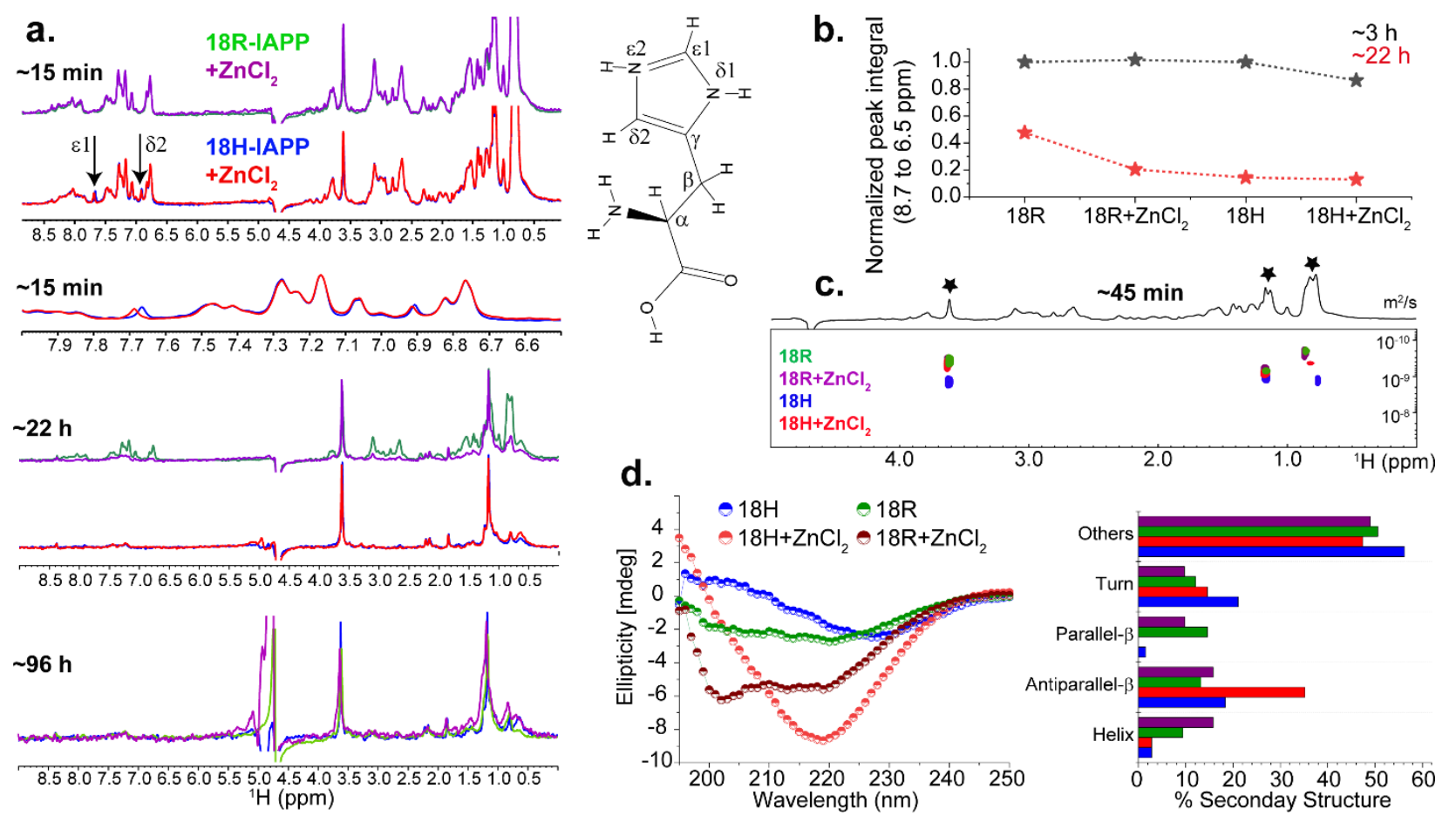

Fig. 5. Monitoring the effect of zinc on wild-type human (18H) and mutant (H18R) IAPP peptides by NMR and CD spectroscopy. a. Time-lapse ${ }^{1} \mathrm{H}$ NMR spectra of $65 \mu \mathrm{M} 18 \mathrm{H}$ (blue and red) and H18R (green and purple) in the absence (blue and green) and presence (red and purple) of 5 molar excess of zinc as indicated. The two peaks for $\varepsilon 1$ and $\delta 2$ protons (molecular structure of histidine and proton labeling are shown on the right) observed in $18 \mathrm{H}$ and absent in H18R-IAPP are indicated by two arrows at $6.90(\delta 2)$ and $7.66(\varepsilon 1) \mathrm{ppm}$. Zinc binding showed a reduction in NMR signal intensity and chemical shift change selectively for the hIAPP sample ( $\sim 3 \mathrm{~h}$ red vs blue spectrum). At a time interval of $\sim 22 \mathrm{~h}$, H18R-IAPP signals were detectable (green) only in the absence of zinc whereas 18H-IAPP incubated with/without zinc showed significant signal loss. At $~ 96 \mathrm{~h}$ all of the IAPP samples showed a complete loss of amideproton $(\mathrm{NH})$ signals. b. The integrated relative change in NMR signal intensities (for the 6.5 to $8.7 \mathrm{ppm}$ region) are plotted for 3 and 22 hours; the signal intensities are normalized with respect to the respective values obtained at $\sim 3 \mathrm{~h}$. c. Plot illustrating the diffusion constants measured from $18 \mathrm{H}$ and $18 \mathrm{R}$ peptide samples incubated for $\sim 3 \mathrm{~h}$ with and without zinc as indicated. The diffusion constants are calculated for a few selected well-resolved peaks (indicated by 
$\star$ ). All NMR samples were prepared using $10 \mathrm{mM}$ Tris-d11, $100 \mathrm{mM} \mathrm{NaCl}, \mathrm{pH} 7.4$ buffer, and the spectra were recorded on a $500 \mathrm{MHz}$ Bruker NMR spectrometer at $25^{\circ} \mathrm{C}$. d. Secondary structure analysis of the IAPP samples used for NMR measurements incubated for $\sim 96 \mathrm{~h}$ using $\mathrm{CD}$ spectroscopy at $25^{\circ} \mathrm{C}$. The $\%$ of different secondary structure shown on the right are calculated using the BestSel program.

The size of molecular species present in the samples was estimated using the Stokes-Einstein equation: $r=\frac{k T}{6 \pi \rho D}$ where $\mathrm{r}$ is the radius of the species, $\mathrm{k}$ is the Boltzmann's constant, $\mathrm{T}$ is sample temperature, $\rho$ is the viscosity and D is the diffusion constant (Nowick et al. 2018). Using the experimentally measured values for $D$ (for the peaks at $\sim 3.6$ and $0.8 \mathrm{ppm}$, given in Table 1), $\mathrm{T}=25{ }^{\circ} \mathrm{C}$, about 3 times increase in the size is estimated for the species present in zinc-containing hIAPP as compared to the zinc-free hIAPP sample. Whereas the zinc-containing H18R-IAPP sample presented nearly no change in the molecular size. It should be noted that any difference in the structure of the IAPP species found in zinc-free versus zinccontaining samples is not taken into account in this estimation, which could alter the estimated size values (Soong et al 2009).

Table 1. Diffusion coefficients $\left(\mathrm{m}^{2} / \mathrm{s}\right)$ of $18 \mathrm{H} / 18 \mathrm{R}-\mathrm{IAPP}$ in the absence and presence of zinc.

\begin{tabular}{|l|l|l|l|l|}
\hline Peak (ppm) & $\mathbf{1 8 H}$ & $\mathbf{1 8 H + Z n C l}$ & \multicolumn{1}{|c|}{$\mathbf{1 8 R}$} & $\mathbf{1 8 R}_{\mathbf{R}} \mathbf{Z n C l}$ \\
\hline$\sim 3.6$ & $1.46 \mathrm{e}-09 \pm 1.5 \mathrm{e}-10$ & $4.59 \mathrm{e}-10 \pm 5.3 \mathrm{e}-11$ & $4.51 \mathrm{e}-10 \pm 5.2 \mathrm{e}-11$ & $3.36 \mathrm{e}-10 \pm 1.4 \mathrm{e}-11$ \\
\hline$\sim 1.1$ & $1.15 \mathrm{e}-09 \pm 8.6 \mathrm{e}-11$ & $8.08 \mathrm{e}-10 \pm 5.0 \mathrm{e}-11$ & $8.10 \mathrm{e}-10 \pm 4.0 \mathrm{e}-11$ & $6.74 \mathrm{e}-10 \pm 4.0 \mathrm{e}-11$ \\
\hline$\sim 0.8$ & $1.23 \mathrm{e}-09 \pm 1.1 \mathrm{e}-10$ & $4.35 \mathrm{e}-10 \pm 3.5 \mathrm{e}-11$ & $2.37 \mathrm{e}-10 \pm 1.6 \mathrm{e}-11$ & $2.20 \mathrm{e}-10 \pm 1.6 \mathrm{e}-11$ \\
\hline
\end{tabular}

At a time-point of 22 -hour, hIAPP in the absence and presence of 5 molar excess $\mathrm{ZnCl}_{2} \mathrm{showed}$ significant line-broadening suggesting an increase in the size of the IAPP species present in the solution. Whereas, at the same time-point, several of the H18R-IAPP proton NMR signals were detectable (Figure 5a) with $\sim 50 \%$ reduction in signal intensity (Figure 5b). Remarkably, H18R-IAPP mixed with 5 molar excess of $\mathrm{ZnCl}_{2}$ showed a significant signal loss similar as compared that observed for hIAPP and hIAPP-ZnCl 2 (Figure 5a, 5b)). These observation suggest that, although zinc binding is specific to 18H in wild-type IAPP, in mutants, zinc could interact with other N-terminal amino acids to drive IAPP aggregation as recently observed for rat IAPP (Magrì et al., 2020). The amide and aromatic proton signals in all NMR samples were found to be undetectable at a time of 96-hour indicating IAPP aggregation.

The secondary structure analyses of both wild-type and H18R-IAPP samples incubated over 96 hours in the absence and presence of zinc were next carried out using CD spectroscopy. It should be noted that all NMR samples at $\sim 96$ hours showed visible aggregates in the solution. As shown in Figure 5d, CD spectrum of $18 \mathrm{H}$-IAPP presented a CD minimum centred at $\sim 227 \mathrm{~nm}$ which in BestSel is characterized with an antiparallel $\beta$-sheet structure (18.4\%). hIAPP mixed with zinc showed a CD minimum cantered at $\sim 218$ $\mathrm{nm}$ that correspond to $35.2 \%$ antiparallel $\beta$-sheet. Interestingly, H18R-IAPP in the presence and absence of zinc presented two CD minima cantered at $\sim 208$ and $\sim 222 \mathrm{~nm}$ indicating an alpha-helical structure. Secondary structure quantification of the H18R-IAPP CD spectrum in the absence of zinc presented 9.4, 13.2 and $14.6 \%$ of $\alpha$-helix, antiparallel and parallel $\beta$-sheets, respectively. The helical structure was found to be more profound (15.8\%) in the presence of zinc as shown in Figure 5d. Taken together, the NMR and CD results indicate zinc binding to hIAPP and H18R-IAPP peptides generate aggregates that are composed of $\beta$ sheet and $\alpha$-helix rich secondary structures.

\subsection{The presence of zinc does not modify IAPP-membrane damage}

To determine the effect of membrane damage on the binding of zinc to wild-type IAPP and the mutant peptides, we performed calcein membrane leakage experiments. Our data indicate that the wild-type hIAPP induces a significant leakage in DOPC/DOPS vesicles to about $85 \%$ of the total vesicles content measured after $24 \mathrm{~h}$ (Fig 6a). The presence of zinc increases this value to $95-100 \%$. For H18R-IAPP and H18KIAPP, a high concentration of zinc (molar ratio of 1:100 hIAPP:zinc) decreases the rate of membrane leakage to approximately $20 \%$ (Fig 6b,c). In contrary, at a high concentration of zinc, the peptide H18E-IAPP, with a negatively charged amino acid, induces more leakage than with a low concentration of zinc (Fig 6d). Zinc seems to not have any specific effect on H18A-IAPP-membrane damage (Fig 6e). Next, the same ThT experiments were carried out in the presence of DOPC/DOPS vesicles and zinc. The effect of zinc on the lag-time extension is shown in Fig 6f,j. For wild-type hIAPP, the presence of zinc does not modify the lagtime of fibril formation (Fig 6f). However, zinc exhibits inhibition capabilities for both H18R-IAPP and H18K-IAPP, increasing the lag-time by a factor of approximately 3.8 and 2.8 , in agreement with the 
membrane leakage experiment. For both H18E-IAPP and H18A-IAPP, the presence of zinc does not change the fibril formation. Our results demonstrate that in the presence of membranes, zinc changes the membrane properties at high concentration but only to the H18R-IAPP and H18K-IAPP peptides. As previously, the samples from the ThT experiments were stained, blotted and observed by TEM. Fig. 6k,o show the images of the peptides in the presence of zinc (ratio 1:100). Zinc does not modify the hIAPP fibrils morphology neither lead to a decrease of the width of the fibrils. For the mutated peptides, zinc causes significant reduction in the amounts of fibrils. In addition, for H18A-IAPP, only very thin fibrils were observed but amorphous aggregates were present in the whole grids. Altogether, our results demonstrate that zinc do not influence hIAPP-membrane fibril formation neither hIAPP-membrane damage, while zinc decreases the rate of hIAPP amyloid formation in solution. Zinc reduced fibril formation and membrane leakage for the peptides H18RIAPP and H18K-IAPP at a high concentration in the presence of membranes.

In the presence of membrane, zinc does not alter hIAPP fibril formation while we saw that in solution zinc slows down the kinetics of fibril formation. Zinc binding to Lys1 and His18, both residues localized in the N-terminal part of hIAPP. We thus hypothesize that these residues might not be accessible to zinc in the presence of membranes as the N-terminal part of hIAPP is known to interact strongly the membranes (Engel et al. 2006, Nanga et al. 2008, Nanga et al. 2009, Nanga et al. 2011). We also note that zinc complexed with EGCG is able to suppress the fibril formation of wild-type IAPP both in solution as well as in the presence of membrane as demonstrated in a recent study (Lee et al. 2019).

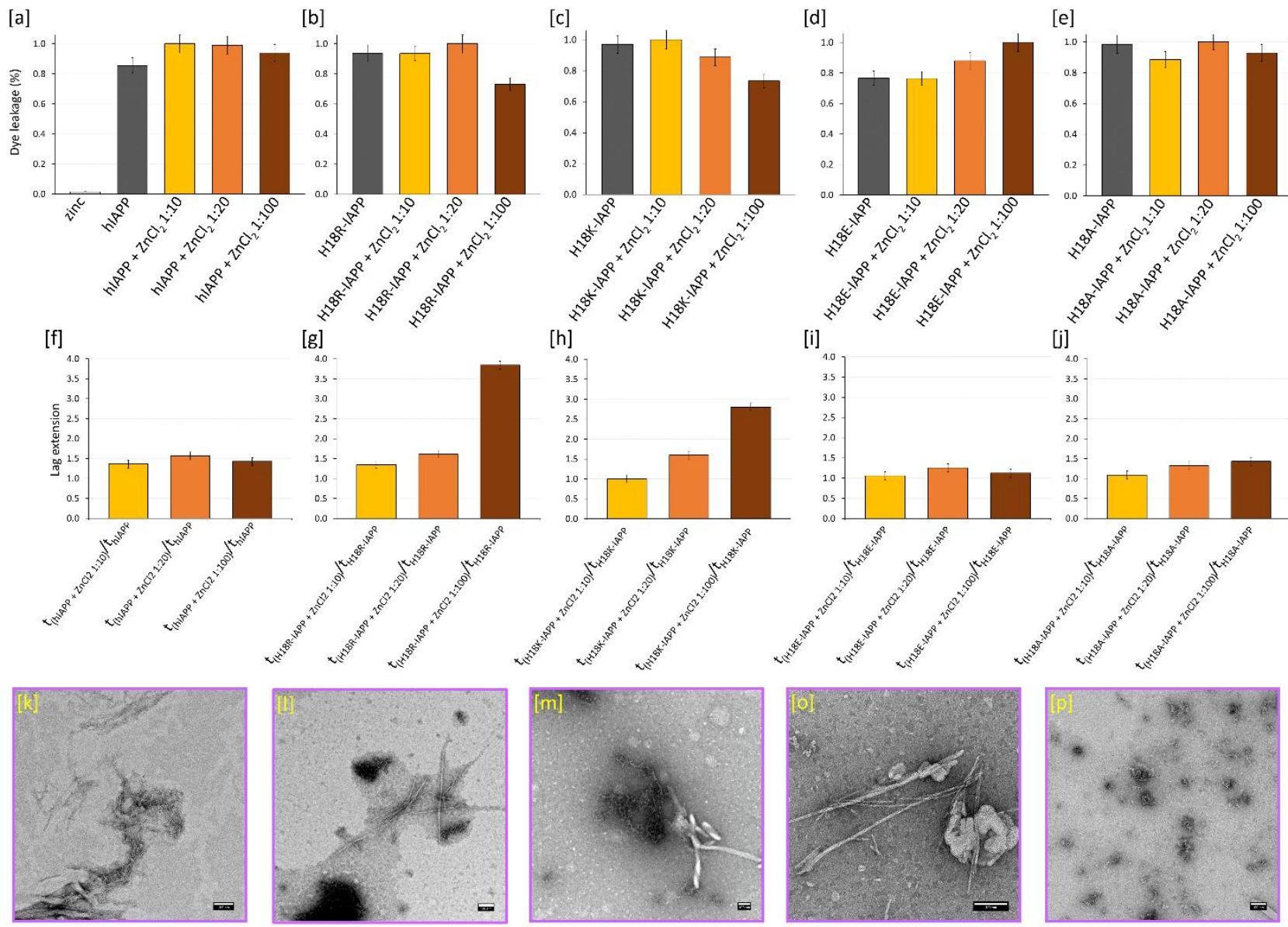

Fig. 6. Effect of zinc on IAPP-membrane damage and IAPP fibril formation at a peptide:lipid ratio of 1:20. (a-e) Maximum of membrane permeabilization for wild-type hIAPP (grey) and the mutant peptides in the presence of zinc measured at $24 \mathrm{~h}$ (ratio 1:5, yellow; 1:20, orange; and 1:100, brown). Zinc alone do not affect the membrane properties (a, light grey). (f-j) Effect of zinc on fibril formation lag-time at the molar ratios 1:5 (yellow), 1:20 (orange) and 1:100 (brown). (k-o) TEM images of hIAPP and the mutant peptides in the presence of zinc (molar ratio 1:100). Scale bars in the TEM images $(k-p)$ indicating $100 \mathrm{~nm}$.

\section{Conclusion}

Insulin and zinc are two main components of the secretory granules. Because hIAPP is stored in the $\beta$-cell granules, it is natural to hypothesize that zinc or insulin could affect hIAPP's fibril formation in vivo. Our 
results reported in this study suggest that insulin strongly affects fibril formation and fibril morphology of IAPP independent of the nature of the residue-18, even at a low ratio of 1:1 insulin:IAPP. However, in the presence of a lipid membrane, insulin inhibits hIAPP fibril formation and hIAPP induced membrane leakage albeit to a lesser extent for the mutant peptides than that observed for the wild-type hIAPP. Our results suggest that the residue-18 is not the only residue that binds to insulin and allows insulin to reduce hIAPP fibril formation. In the presence of zinc, the data demonstrate that there is no one-to one relationship between the kinetics of fibril formation and the concentration of zinc. In solution, the presence of zinc changes the kinetics of fibril formation for the wild-type but not for all the mutant peptides. This probably indicates that zinc favorably binds to the residue histidine-18. In the presence of a lipid membrane, zinc does not seem to alter IAPP fibril formation neither the IAPP-induced membrane leakage even for the wild-type peptide. Based on the results presented in this study and in previous studies (Engel et al. 2006; Brender et al. 2007, Brender et al. 2011), we hypothesis that zinc does not influence hIAPP fibrillation in the presence of a lipid membrane because the N-terminal region of hIAPP (residues 1-19) is involved in the membrane interactions as revealed by the NMR structure (Brender et al 2008) and is therefore not accessible to zinc.

\section{Declaration of Competing Interest}

The authors report no declarations of interest.

\section{Acknowledgements}

The research in the Ramamoorthy lab is supported by NIH (AG048934 to A.R.). This work has benefited from the facilities of UMS3033/US001-IECB.

\section{References}

World Health Organization Fact Sheet-https://www.who.int/health-topics/diabetes\#tab=tab_1

Abedini, A., Raleigh, D.P. 2005. The role of His-18 in amyloid formation by human islet amyloid polypeptide. Biochemistry 2005 44, 16284-16291.

Anguiano, M., Nowak, R.J., Lansbury, P.T., Jr. 2002. Protofibrillar islet amyloid polypeptide permeabilizes synthetic vesicles by a pore-like mechanism that may be relevant to type II diabetes. Biochemistry 41, 11338-11343.

Atrián-Blasco, E., Gonzalez, P., Santoro, A., Alies, B., Faller, P., Hureau, C. 2018. Cu and Zn coordination to amyloid peptides: From fascinating chemistry to debated pathological relevance. Coord. Chem. Rev. 375, 38-55.

Baram, M., Gilead, S., Gazit, E., Miller, Y. 2018. Mechanistic perspective and functional activity of insulin in amylin aggregation. Chem. Sci. 9, 4244-4252.

Bellia, F., Grasso, G. 2014. The role of copper(II) and zinc(II) in the degradation of human and murine IAPP by insulin-degrading enzyme. J Mass Spectrom. 49, 274-279.

Betsholtz, C., Christmansson, L., Engstrom, U., Rorsman, F., Svensson, V., Johnson, K.H. and Westermark, P. 1989. Sequence divergence in a specific region of islet amyloid polypeptide (IAPP) explains differences in islet amyloid format

ion between species. FEBS Lett. 251, 261-264.

Brender, J.R., Dürr, U.H., Heyl, D., Budarapu, M.B., Ramamoorthy, A. 2007. Membrane fragmentation by an amyloidogenic fragment of human Islet Amyloid Polypeptide detected by solid-state NMR spectroscopy of membrane nanotubes Biochim. Biophys. Acta 1768, 2026-2029.

Brender, J.R., Hartman, K., Reid, K.R., Kennedy, R.T., Ramamoorthy, A. 2008. A single mutation in the nonamyloidogenic region of islet amyloid polypeptide greatly reduces toxicity. Biochemistry 47, 1268012688. 
Brender, J.R., Lee, E.L., Cavitt, M.A., Gafni, A., Steel, D.G., Ramamoorthy, A. 2008. Amyloid fiber formation and membrane disruption are separoe processes localized in two distinct regions of IAPP, the type-2-diabetes-related peptide. J. Am. Chem. Soc. 130, 6424-6429.

Brender, J.R., Hartman, K., Nanga, R.P., Popovych, N., de la Salud Bea, R., Vivekanandan, S., Marsh, E.N., Ramamoorthy, A. 2010. Role of zinc in human islet amyloid polypeptide aggregation. J. Am. Chem. Soc. 132, 8973-8983.

Brender, J.R., Lee, E.L., Hartman, K., Wong, P.T., Ramamoorthy, A., Steel, D.G., Gafni, A. 2011. Biphasic effects of insulin on islet amyloid polypeptide membrane disruption. Biophys. J. 100, 685-692.

Brender, J.R., Salamekh, S., Ramamoorthy, A. 2012. Membrane disruption and early events in the aggregation of the diabetes related peptide IAPP from a molecular perspective Acc. Chem. Res. 45, 454462.

Brender, J.R., Krishnamoorthy, J., Messina, G.M., Deb, A., Vivekanandan, S., La Rosa, C., PennerHahn, J.E., Ramamoorthy, A. 2013. Zinc stabilization of prefibrillar oligomers of human islet amyloid polypeptide. Chem Commun. 49, 3339-3341.

Brender, J.R., Krishnamoorthy, J., Sciacca, M.F., Vivekanandan, S., D'Urso, L., Chen, J., La Rosa, C., Ramamoorthy, A. 2015. Probing the sources of the apparent irreproducibility of amyloid formation: drastic changes in kinetics and a switch in mechanism due to micelle like oligomer formation at critical concentrations of IAPP. J. Phys. Chem B. 119, 2886-2896.

Butler, A.E., Janson, J., Bonner-Weir, S., Ritzel, R., Rizza, R.A., Butler, P.C. 2003. Beta-cell deficit and increased beta-cell apoptosis in humans with type 2 diabetes. Diabetes 52, 102-510.

Caillon, L., Duma, L., Lequin, O., Khemtemourian, L. 2014. Cholesterol modulates the interaction of the islet amyloid polypeptide with membranes. Mol. Membr. Biol. 31, 239-249.

Caillon, L., Lequin, O., Khemtémourian, L. 2013. Evaluation of membrane models and their composition for islet amyloid polypeptide-membrane aggregation. Biochim. Biophys. Acta 1828, 20912098.

Clark, A., Wells, C.A., Buley, I.D., Cruickshank, J.K., Vanhegan, R.I., Matthews, D.R., Cooper, G.J., Holman, R.R., Turner, R.C. 1988. Islet amyloid, increased A-cells, reduced B-cells and exocrine fibrosis: quantitative changes in the pancreas in type 2 diabetes. Diabetes Res. 9, 151-159.

Cooper, G.J., Willis, A.C., Clark, A., Turner, R.C., Sim, R.B., Reid, K.B. 1987. Purification and characterization of a peptide from amyloid-rich pancreases of type 2 diabetic patients. Proc. Natl. Acad. Sci. U. S. A. 84, 8628-8632.

Cui, W., Ma, J.W., Lei, P., Wu, W.H., Yu, Y.P., Xiang, Y., Tong, A.J., Zhao, Y.F., L,i Y.M. 2009. Insulin is a kinetic but not a thermodynamic inhibitor of amylin aggregation. FEBS J. 276, 3365-3371.

Despa, S., Margulies, K.B., Chen, L., Knowlton, A.A., Havel, P.J., Taegtmeyer, H, Bers, D.M., Despa, F. 2012. Hyperamylinemia contributes to cardiac dysfunction in obesity and diabetes: a study in humans and rats. Circ Res. 110, 598-608.

Engel, M.F., Yigittop, H., Elgersma, R.C., Rijkers, D.T., Liskamp, R.M., de Kruijff, B., Höppener, J.W., Killian, J.A. 2006. Islet amyloid polypeptide inserts into phospholipid monolayers as monomer. J Mol Biol. 356,783-789.

Engel, M.F., Khemtémourian, L., Kleijer, C.C., Meeldijk, H.J., Jacobs, J., Verkleij, A.J., de Kruijff, B., Killian, J.A., Höppener, J.W. 2008. Membrane damage by human islet amyloid polypeptide through fibril growth at the membrane. Proc. Natl. Acad. Sci. U. S. A. 105, 6033-6038. 
Enoki, S., Mitsukawa, T., Takemura, J., Nakazato, M., Aburaya, J., Toshimori, H., Matsukara, S. 1992. Plasma islet amyloid polypeptide levels in obesity, impaired glucose tolerance and non-insulindependent diabetes mellitus. Diabetes Res. Clin. Pract.15, 97-102.

Erthal, L.C., Marques, A.F., Almeida, F.C., Melo, G.L., Carvalho, C.M., Palmieri, L.C., Cabral, K.M., Fontes, G.N., Lima, L.M. 2016. Regulation of the assembly and amyloid aggregation of murine amylin by zinc. Biophys. Chem. 218, 58-70.

Formby, B., Schmid-Formby, F., Grodsky, G.M. 1984. Relationship between insulin release and 65zinc efflux from rat pancreatic islets maintained in tissue culture. Diabetes 33, 229-34.

Foster, M.C., Leapman, R.D., Li, M.X., Atwater, I. 1993. Elemental composition of secretory granules in pancreatic islets of Langerhans. Biophys J. 64, 525-32.

Ge, X., Kakinen, A., Gurzov, E.N., Yang, W., Pang, L., Pilkington, E.H., Govindan-Nedumpully, P., Chen, P., Separovic, F., Davis, T.P., Ke, P.C., Ding, F. 2017. Zinc-coordination and C-peptide complexation: a potential mechanism for the endogenous inhibition of IAPP aggregation. Chem Commun (Camb). 53, 9394-9397.

Gilead, S., Wolfenson, H., Gazit, E. 2006. Molecular mapping of the recognition interface between the islet amyloid polypeptide and insulin. Angew Chem Int Ed Engl. 45, 6476-6480.

Hoffmann, A.R.F., Saravanan, M.S., Lequin, O., Killian, J.A., Khemtemourian, L. 2018. A single mutation on the human amyloid polypeptide modulates fibril growth and affects the mechanism of amyloid-induced membrane damage. BBA-Biomembranes 1860, 1783-1792.

Hull, R.L., Westermark, G.T., Westermark, P., Kahn, S.E. 2004. Islet amyloid: a critical entity in the pathogenesis of type 2 diabetes. J Clin Endocrinol Metab. 89, 3629-3643.

Ilitchev, A.I., Giammona, M.J., Schwarze, J.N., Buratto, S.K., Bowers, M.T. 2018. Zinc-Induced Conformational Transitions in Human Islet Amyloid Polypeptide and Their Role in the Inhibition of Amyloidosis. J Phys Chem B. 122, 9852-9859.

Jaikaran, E.T.A.S., Nilsson, M.R., Clark, A. 2004. Pancreatic beta-cell granule peptides form heteromolecular complexes which inhibit islet amyloid polypeptide fibril formation. Biochem J. 377, 709-716.

Janciauskiene, S., Eriksson S., Carlemalm, E., Ahrén, B. 1997. B cell granule peptides affect human islet amyloid polypeptide (IAPP) fibril formation in vitro. Biochem Biophys Res Commun. 30, 580-585.

Janson, J., Ashley, R.H., Harrison, D., McIntyre, S., Butler, P.C. 1999. The mechanism of islet amyloid polypeptide toxicity is membrane disruption by intermediate-sized toxic amyloid particles, Diabetes, 48 , 491-498.

Jha, S., Snell, J.M., Sheftic, S.R., Patil, S.M., Daniels, S.B., Kolling, F.W., Alexandrescu, A.T. 2014. pH dependence of amylin fibrillization. Biochemistry 53, 300-310.

Kayed R., Sokolovn Y., Edmondsn B., McIntiren T.M., Miltonn S.C., Halln J.E., Glaben C.G. 2004. Permeabilization of lipid bilayers is a common conformation dependent activity of soluble amyloid oligomers in protein misfolding diseases. J Biol Chem 279:46363-46366.

Ke, P.C., Sani, M.A., Ding, F., Kakinen, A., Javed, I., Separovic, F., Davis, T.P., Mezzenga, R. 2017. Implications of peptide assemblies in amyloid diseases. Chem Soc Rev. 46, 6492-6531. 
Khemtémourian, L., Lahoz Casarramona, G., Suylen, D.P.L., Hackeng, T.M., Meeldijk, J.D., De Kruijff, B., Höppener, J.W.M., Killian, J.A. 2009. Impaired processing of human pro-islet amyloid polypeptide is not a causative factor for fibril formation or membrane damage in vitro. Biochemistry, 48, 10918-10925.

Khemtémourian, L., Doménech, E., Doux, J.P.F., Koorengevel, M.C., Killian, J.A. 2011. Low pH acts as inhibitor of membrane damage induced by human islet amyloid polypeptide. J Am Chem Soc 133, 15598-15604.

Khemtemourian, L., Guillemain, G., Foufelle, F., Killian, J.A. 2017. Residue specific effects of human islet polypeptide amyloid on self-assembly and on cell toxicity. Biochimie 142:22-30.

Knight, J. D., and Miranker, A. D. 2004. Phospholipid catalysis of diabetic amyloid assembly, J. Mol. Biol. 341, 1175-1187.

Knight, J.D., Williamson, J.A., Miranker, A.D. 2008. Interaction of membrane-bound islet amyloid polypeptide with soluble and crystalline insulin. Protein Sci. 17, 1850-1856.

Larson, J.L., Miranker, A.D. 2004. The mechanism of insulin action on islet amyloid polypeptide fiber formation. J Mol Biol. 335, 221-31

Lee, Y.H., Lin, Y., Cox, S.J., Kinoshita, M., Sahoo, B.R., Ivanova, M., Ramamoorthy, A.2019. Zinc boosts EGCG's hIAPP amyloid Inhibition both in solution and membrane. Biochim. Biophys. Acta Proteins Proteom. 1867, 529-536.

Magrì, A., Tabbì, G., Di Natale, G., La Mendola, D., Pietropaolo, A., Zoroddu, M.A., Peana, M., Rizzarelli, E. 2020. Zinc Interactions with a Soluble Mutated Rat Amylin to Mimic Whole Human Amylin: An Experimental and Simulation Approach to Understand Stoichiometry, Speciation and Coordination of the Metal Complexes. Chemistry. 26, 13072-13084.

Marcinkiewicz, M., Ramla, D., Seidah, N.G., Chretien, M. 1994. Developmental expression of the prohormone convertases PC1 and PC2 in mouse pancreatic islets. Endocrinology 135, 1651-1660.

Marzban, L., Rhodes, C.J., Steiner, D.F., Haataja, L., Halban, P.A., Verchere C.B. 2006. Impaired NH2terminal processing of human proislet amyloid polypeptide by the prohormone convertase PC2 leads to amyloid formation and cell death. Diabetes 55, 2192-201.

Micsonai, A., Wien, F., Kernya, L., Lee, Y.H., Goto, Y., Réfrégiers, M., Kardos, J., 2015. Accurate secondary structure prediction and fold recognition for circular dichroism spectroscopy. Proc. Natl. Acad. Sci. U. S. A. 112, E3095-E3103.

Milardi, D., Gazit, E., Radford, S.E., Xu, Y., Gallardo, R.U., Caflisch, A., Westermark, G.T., Westermark, P., La Rosa, C., Ramamoorthy, A., 2021. 2021. Proteostasis of Islet Amyloid Polypeptide: A Molecular Perspective of Risk Factors and Protective Strategies for Type II Diabetes. Chem Rev. 121, 1845-1893.

Mirzabekov, T.A., Lin, M.C., Kagan, B.L. 1996. Pore formation by the cytotoxic islet amyloid peptide amylin. J. Bio.1 Chem. 271, 1988-1992.

Nanga, R.P., Brender, J.R., Xu, J., Veglia, G., Ramamoorthy, A. 2008. Structures of rat and human islet amyloid polypeptide IAPP(1-19) in micelles by NMR spectroscopyBiochemistry 47, 12689-12697.

Nanga, R.P., Brender, J.R., Vivekanandan, S., Ramamoorthy, A. 2011. Structure and membrane orientation of IAPP in its natively amidated form at physiological $\mathrm{pH}$ in a membrane environment. Biochim. Biophys. Acta 1808, 2337-2342. 
Nanga, R.P., Brender, J.R., Xu, J., Hartman, K., Subramanian, V., Ramamoorthy, A. 2019. Threedimensional structure and orientation of rat islet amyloid polypeptide protein in a membrane environment by solution NMR spectroscopy. J. Am. Chem. Soc. 131, 8252-8261.

Nedumpully-Govindan, P., Ding, F. 2015. Inhibition of IAPP aggregation by insulin depends on the insulin oligomeric state regulated by zinc ion concentration. Sci. Rep. 5, 8240.

Nedumpully-Govindan, P., Yang, Y., Andorfer, R., Cao, W., Ding, F. 2015. Promotion or inhibition of islet amyloid polypeptide aggregation by zinc coordination depends on its relative concentration. Biochemistry 54, 7335-7344.

Paulsson, J.F., Westermark, G.T. 2005. Aberrant processing of human proislet amyloid polypeptide results in increased amyloid formation. Diabetes 54, 2117-25.

Porat, Y., Kolusheva, S., Jelinek, R., Gazit, E. 2003. The human islet amyloid polypeptide forms transient membrane-active prefibrillar assemblies. Biochemistry 42,10971-10977.

Quist, A., Doudevski, I., Lin, H., Azimova, R., Ng, D., Frangione, B., Kagan, B., Ghiso, J., Lal, R. 2005. Amyloid ion channels: A common structural link for protein misfolding disease. Proc. Natl. Acad. Sci. USA 102, 10427-10432.

Rodriguez Camargo, D.C., Korshavn, K.J., Jussupow, A., Raltchev, K., Goricanec, D., Fleisch, M., Sarkar, R., Xue, K., Aichler, M., Mettenleiter, G., Walch, A.K., Camilloni, C., Hagn, F., Reif, B., Ramamoorthy, A. 2017. Stabilization and structural analysis of a membrane-associated hIAPP aggregation intermediate. Elife 6, e31226.

Rouser, G., Fkeischer, S., Yamamoto, A. 1970. Two dimensional then layer chromatographic separation of polar lipids and determination of phospholipids by phosphorus analysis of spots. Lipids 5, 494-496.

Sahoo, B.R., Genjo, T., Nakayama, T.W., Stoddard, A.K., Ando, T., Yasuhara, K., Fierke, C.A., Ramamoorthy, A., 2019. A cationic polymethacrylate-copolymer acts as an agonist for $\beta$-amyloid and an antagonist for amylin fibrillation. Chem. Sci. 10, 3976-3986.

Salamekh, S., Brender, J.R., Hyung, S.J., Nanga, R.P., Vivekanandan, S., Ruotolo, B.T., Ramamoorthy, 2011. A two-site mechanism for the inhibition of IAPP amyloidogenesis by zinc. J. Mol. Biol. 410, 294306.

Salazar Vazquez, L.S., Blondeau, B., Cattan, P., Armanet, M., Guillemain, G., Khemtemourian, L. 2020. The flanking peptides issue from the maturation of the human islet amyloid polypeptide (hIAPP) slightly modulate hIAPP-fibril formation but not hIAPP-induced cell death. Biochimie, 170, 26-35.

Sciacca, M.F., Brender, J.R., Lee, D.K., Ramamoorthy, A. 2012. Phosphatidylethanolamine enhances amyloid fiber-dependent membrane fragmentation. Biochemistry 51, 7676-7684.

Sellin, D., Yan, L.M., Kapurniotu, A., Winter, R. 2010. Suppression of IAPP fibrillation at anionic lipid membranes via IAPP-derived amyloid inhibitors and insulin. Biophys Chem. 150, 73-79.

Soong, R., Brender, J.R., Macdonald, P.M., Ramamoorthy, A. 2009. Association of highly compact type II diabetes related islet amyloid polypeptide intermediate species at physiological temperature revealed by diffusion NMR spectroscopy. J. Am. Chem. Soc. 131, 7079-7085.

Taylor, C.G. 2005. Zinc, the pancreas, and diabetes: insights from rodent studies and future directions. Biometals 18, 305-312.

Tian, H., Wang, Z.Y. 2019. Zinc Chelator Inhibits zinc-induced islet amyloid polypeptide deposition and apoptosis in INS-1 Cells. Biol. Trace Elem. Res. 189, 201-208. 
Wang, H. Raleigh, D.P. 2014. The ability of insulin to inhibit the formation of amyloid by pro-islet amyloid polypeptide processing intermediates is significantly reduced in the presence of sulfated glycosaminoglycans. Biochemistry. 53, 2605-2614

Wei, L., Jiang, P., Yau, Y.H., Summer, H., Shochat, S.G., Mu, Y., Pervushin, K. 2009. Residual structure in islet amyloid polypeptide mediates its interactions with soluble insulin. Biochemistry. 48, 2368-2376.

Westermark, P., Engstrom, U., Johnson, K.H., Westermark, G.T. and Betsholtz, C. 1990. Islet amyloid polypeptide - pinpointing amino-acid-residues linked to amyloid fibril formation. Proc. Natl. Acad. Sci. U.S.A. 87, 5036-5040.

Westermark, P., Li, Z.C., Westermark, G.T., Leckström, A., Steiner, D.F. 1996. Effects of beta cell granule components on human islet amyloid polypeptide fibril formation, FEBS Lett. 379, 203-206.

Westermark, P., Wernstedt, C., Wilander, E., Hayden, D.W., O’Brien, T.D., Johnson, K.H. 1987. Amyloid fibrils in human insulinoma and islets of Langerhans of the diabetic cat are derived from a neuropeptidelike protein also present in normal islet cells. Proc Natl Acad Sci U S A. 84, 3881-3885.

Wang, Y., Truex, N.L., Vo, N.D.P., Nowick, J.S. 2018. Effects of charge and hydrophobicity on the oligomerization of peptides derived from IAPP. Bioorg. Med. Chem. 26, 1151-1156.

Wiltzius, J.J., Sievers, S.A., Sawaya, M.R., Eisenberg, D. 2009. Atomic structures of IAPP (amylin) fusions suggest a mechanism for fibrillation and the role of insulin in the process. Protein Sci. 18, 15211530 .

Wineman-Fisher, V., Miller, Y. 2016. Effect of $\mathrm{Zn}(2+)$ ions on the assembly of amylin oligomers: insight into the molecular mechanisms. Phys. Chem. Chem. Phys. 18, 21590-21599.

Wineman-Fisher, V., Miller, Y. 2017. Insight into a New Binding Site of Zinc Ions in Fibrillar Amylin. ACS Chem Neurosci. 8, 2078-2087

Yamaguchi, T., Matsuzaki, K., Hoshino, M., 2011. Transient formation of intermediate conformational states of amyloid- $\beta$ peptide revealed by heteronuclear magnetic resonance spectroscopy. FEBS Lett. 585, 1097-1102. 